\title{
What Determines the Resistance and the Tilt of an Aeroplane ${ }^{1}$
}

\section{By Sir Joseph LARMor, F.R.S.}

NE used to recognise that the exigencies of flight in the tenuous air prescribed a limit to the bulk of a bird, as compared for example with a whale. Yet nowadays every day loads of twenty tons of stuff or possibly far more are carried over long journeys, owing to the power available, solely on wings. How does the attenuated aerial medium find means of supporting such an astonishing mass? To experts the fact is familiar, and so scarcely demands explanation. Indeed, the source of the support in plain terms is just as wonderful as the fact itself. The load is held up solely by the swirl that it produces and leaves behind, and this vertical support must be the only dynamical effect of the swirl when the speed is steady : there remains the question how precisely this result is adjusted. Unfortunately the wakes from screw and wings can scarcely be additive without some mutual interference, though momenta are additive always. For example the spread of the wings is adapted readily to counteract the rotational grip of the screw. Stability is theoretically (G. H. Bryan) another affair.

Whether the supporting medium is air, or water, or even pitch, provided only it is of uniform density everywhere, the momentum, with which the flight is concerned, proves to be expressible at each instant in terms of the distribution of swirl or vorticity alone. Force is experienced by the travelling system equal and opposite to the rate at which momentum is shed away into the wake of its motion. The nature of the swirl passing into the wake thus determines all. In the ideal perfect fluid of abstract hydrodynamics there would be no wake, and therefore no force affecting the translatory motion, though the mass may twirl in permanent precessional spin. If this train of ideas is

1 Abstracted, with additions, from Proc. Cambridge Phil. Soc., Feb. 1, 1927, pp. 617-630. right it is impossible for a circulation round the wings of an aeroplane to sustain it, except in so far as it has to be associated with a vortical wake.

The formula for the momentum associated with each element of whirl in the ambient medium, of whatever kind it be and however complex its internal friction, provided only it is of uniform density, turns out to be unexpectedly simple. There is translational momentum equal, as applied at any chosen origin, to the vector moment of the mass-vorticity of the element (mass multiplied by spin), combined with rotational momentum around that origin equal to this massvorticity multiplied by the square of the distance with sign reversed. Now vorticity has the advantage of being a quality of considerable persistence, unless the internal friction is high : if then this field of spin could be sufficiently explored by observation, it would only be necessary, in order to obtain the forces operating, to trace out the rate at which the derived system of momentum thus associated with the travelling machine is changing. Many special illustrations present themselves. For example, a travelling aeroplane adjusts its presentation so that this moment of momentum, with regard to the point where the pull of the screw intersects the line of weight, is not subject to loss into the wake : such automatic adjustment would tend to nip together the two boundary sheets of the vortical trail, which thus would open out only at the ends of the wings. Again, a windmill parachute appears to be more effective than the simple umbrella type : if so, the cause doubtless declares itself in a wind-channel by the contrasted types of whirl in the wakes they leave behind. And generally, the performance of any propelling screw wholly submerged would be determinable in terms of the whirl in its wake alone, if only that could be explored.

\section{The Wren-Ashmole-Plot Memorial Windows at Oxford.}

THE public unveiling of the memorial windows to 1 Sir Christopher Wren, Ashmole, and Dr. Plot by the Chancellor of the University, Lord Cave, took place at Oxford on May 17 in ideal circumstances of weather, after the ceremony of the presentation of honorary degrees to MM. Doumergue and Briand. Speeches were delivered in the Divinity School by the representatives of the bodies who have given the windows. Mr. Madan, on behalf of Brasenose College, spoke on Ashmole as the founder of the oldest museum of natural history ; Mr. Guy Dawber, president of the Royal Institute of British Architects, pronounced an éloge on Wren ; and the Public Orator, Mr. Poynton, representing University, Magdalen, and Hertford Colleges, made a witty speech on Dr. Plot. The windows are a notable addition to the beauty of the staircase of the Old Ashmolean Museum, and will recall to generations of visitors the great pioneer work of this interesting group of men of science of the seventeenth century, some of whose work is illustrated in the Lewis Evans Collection on the upper floor of the building. The Chancellor expressed the grateful thanks of the University to the respective donors. $\mathrm{He}$ also paid a well-deserved tribute to Dr. R. T. Gunther, the Curator of the Lewis Evans Collection of Scientific Instruments, to whose zeal and energy are to be attributed the excellent arrangement and appropriate housing of the Lewis Evans Collection, and at whose instigation the donors of the memorial windows were moved to undertake these admirable additions to the interest of the historic building that contains them.

The new windows in the Old Ashmolean Building commemorate the work of Ashmole and his three friends, whose collective scientific achievements during the second half of the seventeenth century have proved second to none even in that fertile period of English science. The word collective is used designedly, for it is extremely probable that the labour or good intention of any one member of the group would have been of no avail without the faithful co-operation of the others. They have one and all in their several ways participated in the establishment in Oxford of the first public museum of natural history in Britain. It is meet that so great a public service should be recognised in the building, which fortunately is still standing, a monument to their great work.

The oldest member of the group, John Tradescant, was a great collector, a scientific traveller, a pioneer who introduced new plants into Europe, and followed his father as the owner of the first London museum, at Lambeth. To him succeeded Elias Ashmole, a great transmitter. He realised the supreme educational value of the collection, and on the death of Tradescant, saved it from being scattered by his widow. His social prestige assured its appreciative acceptance by the University, which received the gift with royalty and a banquet, after the expending of a great sum on a building which is not only the finest classical building

No. 3004, VoL. 119] 